\title{
Perfil antropométrico e funcional de velejadores da classe "Optimist"
}

CDD. 20.ed. 796.033

797.14

\author{
Liliam Fernandes de OLIVEIRA* \\ Daniele POLATO* \\ Rafael Bittencourt ALVES* \\ Suyanne FRAGA* \\ Adriana Ribeiro de MACEDO**
}

*Universidade Federal

do Rio de Janeiro.

**|nstituto Federal de

Educação, Ciência e

Tecnologia do Rio de Janeiro.

\begin{abstract}
Resumo
Este estudo apresenta uma análise descritiva das caracteristicas antropométricas e funcionais de crianças e adolescentes velejadores da classe "Optimist", participantes do campeonato estadual do Rio de Janeiro, em 2006. Foram avaliados 50 velejadores do sexo masculino, sendo 17 da categoria infantil e 33 da juvenil. Os velejadores da categoria infantil apresentaram (valores médios \pm desvio padrão) $152,8 \pm 5,8 \mathrm{~cm}$ de estatura, 45,6 $\pm 5,5 \mathrm{~kg}$ de massa corporal, indice de massa corporal (IMC) de 19,6 $\pm 2,8$ e percentual de gordura de $23,9 \pm 7,0 \%$. Quanto às características funcionais, observou-se força de preensão média de $22,5 \pm 2,9 \mathrm{kgf}$ e salto vertical com altura de $25,5 \pm 4,8 \mathrm{~cm}$. A categoria juvenil apresentou estatura média de $161,7 \pm 7,7 \mathrm{~cm}$, massa corporal de $48,9 \pm 7,5 \mathrm{~kg}$, IMC de $18,7 \pm 2,2 \mathrm{~kg} / \mathrm{m}^{2}$, percentual de gordura de $18,6 \pm 5,5 \%$, força de preensão de $29,6 \pm 5,5 \mathrm{kgf}$ e altura do salto vertical de $31,6 \pm 4,7 \mathrm{~cm}$. A categoria juvenil apresentou estatura e força significativamente maiores que a infantil $(p<0,05)$. Todavia, com 0 percentual de gordura foi significativamente menor, o que resultou em valores de Índice de Massa Corpórea (IMC) e massa corporal similares. Os resultados apontam para um perfil longilíneo dos velejadores juvenis, onde maior força e estatura são acompanhados por redução do percentual de gordura e manutenção da massa corporal, não prejudicando o desempenho do atleta no tipo de embarcação da classe "Optimist".
\end{abstract}

UnItermos: Antropometria; "Optimist"; Crianças; Adolescentes; Velejadores.

\section{Introdução}

Características antropométricas e fisiológicas de atletas de elite são estudadas, visando estabelecer parâmetros para a detecção de talentos e a identificação e elaboração de programas de treinamento mais adequados (ReILlY, BANGSBO \& Franks, 2000). Para Damasgatrd, Bencke, Matthiesen, Petersen e Mülller (2001) é importante também determinar o perfil de crianças e adolescentes competidores, pois a falta de dados morfológicos de atletas adolescentes pode comprometer a seleção de talentos quando ainda jovens (Viviani, Casagrande \& Toniutto, 1993).

Há décadas, estudos antropométricos são conduzidos, em crianças e adolescentes, a fim de caracterizar o perfil do atleta em esportes como a natação (Helmuth, 1980) e o futebol (CACCiari, Mazzanti, Tassinari, Bergamaschi, Magnani, Ghini, Tani,
Drago, Nanni \& Cobianchi, 1989). Helmuth (1980) observou que os nadadores de ambos os sexos são maiores, mais pesados e apresentavam ombros mais largos que as crianças norte-americanas de mesma idade e sexo. CACCIARI et al. (1989) não constataram diferenças significativas na antropometria e maturação biológica entre praticantes e não praticantes de futebol americano competitivo na fase pré-puberal. Na puberdade, entretanto, os atletas se apresentavam mais altos e com maturação biológica mais avançada com relação ao grupo controle.

Alguns estudos posteriores utilizaram parâmetros antropométricos e funcionais, visando comparar atletas de diferentes esportes. LEONE, LARIVIERE e ComTois (2002) observaram que adolescentes atletas do sexo feminino, praticantes de quatro diferentes 
esportes (tênis, natação, patinação no gelo e voleibol), apresentavam características próprias, capazes de discriminá-las em relação ao esporte praticado. A partir de suas características antropométricas e biomotoras, 885 das atletas foram corretamente classificadas. DAMASGAARD et al. (2001) também obtiveram diferenças na antropometria e composição corporal crianças e adolescentes que competiam em diferentes esportes, tendo considerados competidores de tênis, handebol, natação e ginástica. WATTS, JoubERT, LisH e WiLKIns (2003) sabendo as características antropométicas e funcionais de escaladores de elite, avaliaram a presença ou não de tais características de competidores juniores. Observaram, nos competidos juniores, assim como nos de elite, predomina baixa estatura, baixa massa corporal, baixa soma de dobras cutâneas, alta força de preensão, um tipo corporal mais linear e com ombros estreitos em relação ao quadril.

Recentemente, TAn e Sunarja (2007) estudaram 65 velejadores infanto-juvenis. Contudo, o objetivo dos autores foi observar as alteraçóes de massa

\section{Materiais e método}

Cinquenta velejadores do sexo masculino participaram do estudo. Destes, 17 pertenciam à categoria infantil (11-12 anos) e 33 à categoria juvenil (13-14 anos). Todos os 50 atletas participaram do Campeonato Estadual de Vela da classe "Optimist", em novembro de 2006, realizado na cidade do Rio de Janeiro.

O presente estudo foi aprovado pelo Comitê de Ética e Pesquisa do Hospital Universitário Clementino Fraga Filho. A coleta de dados foi realizada antes das competições. Após concordância dos responsáveis, os voluntários foram informados dos procedimentos e finalidades da pesquisa e responderam a um questionário contendo informações referentes ao tempo de prática, volume de treinamento semanal e influência na escolha do esporte. Posteriormente, os atletas foram submetidos aos testes funcionais de salto vertical e "handgrip" e a medidas antropométricas de massa corporal $(\mathrm{kg})$, estatura $(\mathrm{cm})$ e dobras cutâneas $(\mathrm{mm})$, a partir das quais foram estimados o índice de massa corporal $\left(\mathrm{kg} / \mathrm{m}^{2}\right)$ e o percentual de gordura dos voluntários.

O índice de massa corporal (IMC) foi considerado como o quociente da massa corporal pela estatura ao quadrado.O percentual de gordura $(\% \mathrm{G})$ foi estimado a partir de mediçõos de dobras cutâneas $(\mathrm{mm})$ utilizando compasso de dobra cutânea Cercof Ltda, com resolução de $0,1 \mathrm{~mm}$. Para a estimativa, foram corporal e o consumo de carboidratos e fluidos durante um dia de competições, e não caracterizar o perfil antropométrico e funcional dos competidores.

As características antropométricas e funcionais de crianças e adolescentes atletas de esportes em ascensão e menos difundidos, como o iatismo e a escalada, são pouco estudadas. O iatismo vem se difundindo no Brasil, graças aos resultados obtidos em competições de nível internacional. Este ocupa o primeiro lugar, dentre os esportes, em número de medalhas olímpicas. Apresenta como resultados gerais 14 medalhas olímpicas, 55 medalhas em Pan-Americanos e 77 medalhas em campeonatos mundiais (Federação Brasileira de Vela e Motor, 2008). A classe "Optimist" corresponde ao primeiro estágio de aprendizagem da vela e utiliza uma embarcação que suporta apenas um velejador, com idade limite de 15 anos. Sendo assim, o objetivo deste estudo foi traçar e comparar o perfil antropométrico e funcional de crianças e adolescentes do sexo masculino, velejadores da classe "Optimist".

consideradas as dobras das regiōes sub-escapular (SE) e triciptal (TR) do lado direito do corpo, através da equação de Boileau, Lohman e Slaugter (1985) para crianças e jovens do sexo masculino (Equação 1):

$$
\% \mathrm{G}=1,35(\mathrm{TR}+\mathrm{SE})-0,012(\mathrm{TR}+\mathrm{SE}) 2-4,4
$$

A avaliação funcional consistiu no teste de salto vertical (AlmUZAinI, 2007), relacionado com a potência de membros inferiores (Geladas, Nassis \& PaVliceVIC, 2005) e no "handgrip", teste de força de preensão manual (AlmUZAINI, 2007; WATTS et al., 2003).

Para o teste de salto vertical foi utilizada uma plataforma de salto Jump System 1.0 (Cefise Ltda). $\mathrm{O}$ atleta se posicionava descalço, sobre a plataforma, com os membros inferiores em extensão e as mãos no tronco, na altura da cintura pélvica, para evitar a utilização dos membros superiores durante o salto. Após comando verbal específico, o voluntário executava o salto, visando atingir a máxima altura possível. Os momentos de descarga e carga da massa corporal, devidos, respectivamente, à saída e retorno dos pés do voluntário à plataforma foram usados para o cálculo da altura do salto. Foram realizados dois saltos, com intervalo de um minuto entre eles. Foi considerado como resultado final o maior valor, em centímetros. 
No teste de força de preensão foi utilizado um dinamômetro resistivo de preensão manual (Jamar, Sammons Preston Inc.), com empunhadura variável. $\mathrm{O}$ indivíduo era orientado a realizar a força de preensão manual máxima, com o membro superior dominante, mantendo-o ao longo do corpo. Antes de começar o teste, foi feita a adaptação ao instrumento. A adaptação consistiu em uma série de instruções sobre o funcionamento do equipamento, seguida de uma tentativa por parte do voluntário. Após a adaptação, foi solicitado que o indivíduo apertasse com força a empunhadura e soltasse logo em seguida. Foi registrada a força de preensão em kgf.

Foi feita a análise descritiva das variáveis nos grupos infantil e juvenil e, visando avaliar a presença de diferenças entre os valores obtidos em cada uma das categorias, para os diferentes parâmetros considerados, foi aplicado o teste" $t$ " de "Student" para amostras independentes. O aplicativo utilizado foi o de Statistica 5.1 (StatSoft, Inc.). Foi considerado como nível de significância $\alpha<0,05$.

\section{Resultados}

A TABELA 1 apresenta os valores (média \pm desvio padrão) das variáveis massa corporal, altura, índice de massa corporal e percentual de gordura para as categorias infantil e juvenil.

A TABELA 2 apresenta os resultados Média ( \pm desvio padrão) da avaliação funcional dos atletas das categorias juvenil (J) e infantil (I).

A TABELA 3 apresenta informaçôes sobre a idade atual, o tempo de prática da vela e a frequência de treinamento dos atletas das categorias infantil (I) e juvenil (J).

A FIGURA 1 apresenta a presença de parentes que velejem na família dos competidores juvenis e infantis.

TABELA 1 - Média (desvio padrão) de massa corporal, altura, índice de massa corporal (IMC) e percentual de gordura (\%G) dos atletas das categorias infantil (I) ejuvenil (J ).

\begin{tabular}{lcc}
\hline \multicolumn{1}{c}{ Variáveis antropométricas } & $\mathbf{J}(\mathbf{N}=\mathbf{3 3})$ & $\mathbf{I}(\mathbf{N}=\mathbf{1 7})$ \\
\hline Massa corporal $(\mathrm{kg})$ & $48,9( \pm 7,5)$ & $45,6( \pm 5,5)$ \\
Altura $(\mathrm{cm})$ & $161,7( \pm 7,7)$ & $152,8( \pm 5,8)^{*}$ \\
$\mathrm{IMC}\left(\mathrm{kg} / \mathrm{m}^{2}\right)$ & $18,7( \pm 2,2)$ & $19,6( \pm 2,8)$ \\
$\% \mathrm{G}$ & $18,6( \pm 5,5)$ & $23,9( \pm 7,0)^{*}$ \\
\hline
\end{tabular}

TABELA 2 - Média ( \pm desvio padrão) da avaliação funcional dos atletas das categorias juvenil (J) e infantil (I).

\begin{tabular}{lcc}
\hline \multicolumn{1}{c}{ Variáveis funcionais } & $\mathbf{J}(\mathbf{N}=\mathbf{3 3})$ & $\mathbf{I}(\mathbf{N}=\mathbf{1 7})$ \\
\hline Força $(\mathrm{kgf})$ & $29,6( \pm 5,5)$ & $22,5( \pm 2,9)^{*}$ \\
Salto $(\mathrm{cm})$ & $31,6( \pm 4,7)$ & $25,5( \pm 4,7)^{*}$ \\
\hline
\end{tabular}

TABELA 3 - Média ( \pm desvio padrão) do tempo de prática da vela e da frequência semanal de treinamento dos 50 atletas das categorias infantil (I) e juvenil (J).

\begin{tabular}{lclc}
\hline Categorias & Faixa etária da categoria & Tempo de prática & Frequência de treinamento \\
\hline $\mathrm{J}(\mathrm{N}=33)$ & 13 a 14 anos & $4,13( \pm 1,31)$ anos & $3,06( \pm 1,34)$ dias/semana \\
$\mathrm{I}(\mathrm{N}=17)$ & 11 a 12 anos & $3,59( \pm 1,21)$ anos & $3,06( \pm 0,67)$ dias/semana \\
\hline
\end{tabular}


Juvenil

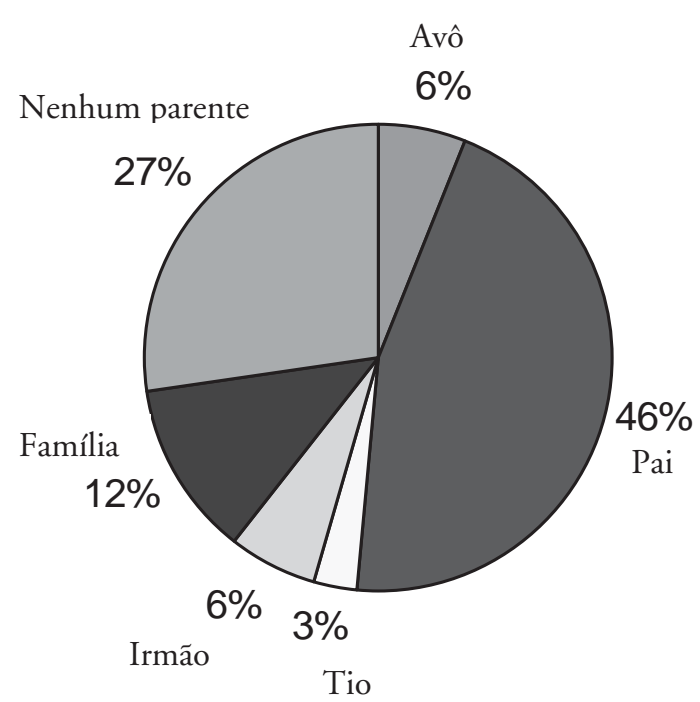

Infantil

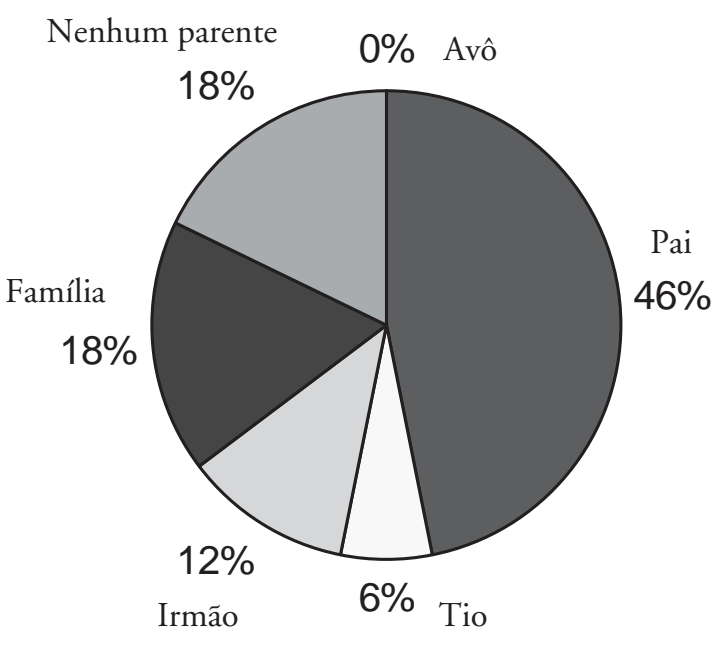

FIGURA 1- Percentual de competidores das categorias J uvenil e Infantil que possuem familiares próximos praticantes devela. A categoria família refere-sea presença demais deum membro dafamília queveleje.

\section{Discussão}

Não foram encontrados, na literatura, estudos similares ao desenvolvido, considerando crianças e adolescentes velejadores da classe "Optimist". Pouco se conhece sobre a prática esportiva e o desenvolvimento da criança velejadora, apesar do esporte de alto rendimento necessitar de que os fundamentos inerentes a ele sejam desenvolvidos desde a infância (BöHME, 2000).

No Brasil, estudos biomecânicos em velejadores são reportados em adultos, nas classes olímpicas e pan-americanas (Roesler, Haupenthal, Schutz \& Menezes, 2005). Em crianças e adolescentes, as alterações de massa corporal e a ingestão nutricional de velejadores da classe "Optimist", durante um dia de competiçôes, foi estudada recentemente no exterior (TAN \& SunARJa, 2007). Os autores encontraram um valor médio de massa corporal de $37,2 \mathrm{~kg}$, inferior à encontrada no presente estudo, para uma população com idade média de 11,6 anos. Todavia, com um desvio padrão bem superior $(8,2$ $\mathrm{kg}$ ), indicando uma maior dispersão dos valores da amostra, em relação ao estudo ora apresentado.

Observou-se neste estudo que a maioria das crianças e adolescentes têm influência familiar, em especial do pai, na escolha da vela como esporte (FIGURA 1) e que ingressam no esporte ainda na infância, com cerca de oito a 10 anos (TABELA 3). $\mathrm{O}$ treinamento dos atletas velejadores participantes do Campeonato Estadual do Rio de Janeiro de 2006 é de, em média, três vezes por semana. Todavia, a moda é de duas vezes por semana. Em relação aos dados antropométricos, o presente estudo descreve que os velejadores da categoria juvenil apresentam maior estatura e um menor percentual de gordura em relação aos da categoria infantil. Apesar de mais altos, o menor $\% \mathrm{G}$, resulta em pesos e, conseqüentemente, em IMC similares. Na prática, observase que jovens, de 13 a 15 anos, mais longilíneos permanecem velejando na classe "Optimist". É comum constatar a migração do adolescente para outras classes, tais como o Laser, quando o velejador adquire uma compleição física mais mesomorfa, desvantajosa para o desempenho em ventos de intensidade baixa ou média. No esporte estudado, o velejador geralmente apresenta melhor desempenho em intensidades de ventos específicas, favorecidas pelo seu biótipo. Contudo, acredita-se que o ideal seja existir um compromisso entre estatura, massa corporal e composição corporal, de forma a possibilitar bom desempenho em diferentes condições de vento. Estudos antropométricos e funcionais, inclusive contendo um maior número de variáveis descritivas, devem ser conduzidos visando a comparação do biótipo e das diferenças funcionais entre as diferentes classes e dentro da mesma classe. 
Na prática da vela, a alta estatura pode dificultar manobras como as cambadas e jibes, causando importante estresse na articulação do joelho, que permanece flexionada a maior parte do tempo. O trabalho de Prestes, Leite, Leite, Donatto, Urtado, Bartolomeu Neto e Dourado (2006) apresenta valores de massa corporal $(\mathrm{I}=53,1 \mathrm{~kg}, \mathrm{~J}=66,1 \mathrm{~kg})$, estatura $(\mathrm{I}=$ $1,61 \mathrm{~m}, \mathrm{~J}=1,74 \mathrm{~m})$ e IMC $\left(\mathrm{I}=20,36 \mathrm{~kg} / \mathrm{m}^{2}, \mathrm{~J}=21,91\right.$ $\mathrm{kg} / \mathrm{m}^{2}$ ) de atletas brasileiros, nadadores das categorias infantil (I) e juvenil (J). SCHNEIDER e Meyer (2005) também relatam valores de massa corporal $(56 \mathrm{~kg})$, altura $(168 \mathrm{~cm}), \operatorname{IMC}\left(19,6 \mathrm{~kg} / \mathrm{m}^{2}\right)$ e $\%$ G $(13,6 \%)$ de jovens nadadores brasileiros do sexo masculino com idade de 13,6 ( $\pm 1,2)$ anos, compatível com a idade de velejadores juvenis. Os valores de altura, massa e índice de massa, relatados nos dois estudos, são superiores aos encontrados no presente estudo. Todavia, com percentual de gordura menor. Tais resultados indicam diferenças no biótipo de nadadores e velejadores brasileiros, provavelmente condizentes com as necessidades da modalidade esportiva praticada.

Em concordância com o presente trabalho, o estudo de Prestes, Leite, Leite, Donatto, Urtado, Bartolomeu Neto e Dourado (2006) obteve maior $\% \mathrm{G}$ na categoria infantil que na juvenil. SCHNEIDER e MeYER (2005) também observaram menor percentual de gordura nos atletas, homens e mulheres, na fase pré-púbere que na púbere. Os dados obtidos para a fase pré-pubere no estudo de SCHNeIDER e Meyer (2005) não foram considerados para no presente estudo devido à menor idade dos nadadores 9,0 ( \pm $0,7)$ em relação aos velejadores da classe infantil.

Os atletas nadadores, em ambos os estudos citados, apresentam maior IMC e menor percentual de gordura, mensurado através de dobras cutâneas, provavelmente devido ao aumento da massa corporal direcionado à maior produção de força para o nado. O padrão antropométrico dos nadadores é caracterizado pelo aumento da massa muscular, principalmente na região torácica e nos membros superiores (Prestes et al., 2006; Schneider e MEYER, 2005), contrastando com o biótipo longilíneo observado no velejador da classe "Optimist".

Baxter-Jones, Helms, Maffulli, Baines-Preece e PREECE (1995) comparou medidas antropométricas de atletas britânicos de diferentes modalidades. Os valores de peso $(51,3 \mathrm{~kg})$ e altura $(161,6 \mathrm{~cm})$ dos nadadores aos 11 anos possuem a mesma tendência observada dos nadadores brasileiros em relação aos velejadores estudados. Os autores encontraram menor estatura para os ginastas $(151,7 \mathrm{~cm})$ e jogadores de futebol $(158,7 \mathrm{~cm})$, em relação aos nadadores.
O perfil dos velejadores se assemelha ao dos escaladores (altura $=162,2 \mathrm{~cm}$, massa $=51,5 \mathrm{~kg}$, IMC $\left.=19,1 \mathrm{~kg} / \mathrm{m}^{2}\right)$, considerando uma idade média um pouco superior da amostra de escaladores $(13,7 \pm$ $2,7)$ anos, no estudo de WatTs et al. (2003). Todavia, o \%G dos escaladores juvenis é inferior (11\%). Os autores afirmam que o escalador de elite adulto apresenta baixa estatura e peso corporal e muito baixo percentual de gordura, tendo biotipo longilíneo com ombros estreitos em relação ao quadril.

O estudo realizado por Farias e SALVAdor (2005), em estudantes brasileiros não atletas, apresentou valores médios de massa corporal (44,2 kg) e estatura $(1,49 \mathrm{~m})$ inferiores aos encontrados neste estudo para as crianças da categoria infantil, enquanto os adolescentes com idades similares à categoria juvenil apresentaram valores de massa corporal $(54,1$ $\mathrm{kg})$ e estatura $(1,63 \mathrm{~m})$ superiores. Tais resultados podem indicar a melhor adequação de adolescentes com menor estatura e peso na classe "ptimist". Tendência inversa foi obtida na comparação com um grupo de meninos adolescentes $(12,3 \pm 0,5$ anos, $38,1 \mathrm{~kg}, 144,4 \mathrm{~cm}$ de altura) sauditas não atletas (AlmuZaini, 2007), com idade compatível com a da categoria juvenil de velejadores. Estes apresentaram menor estatura e peso que os velejadores estudados. Contudo, neste caso, pode ter havido influência da nacionalidade nos resultados.

Quanto às características funcionais avaliadas no presente estudo, foram observadas diferenças entre os velejadores juvenis e infantis. A força de preensão e a impulsão vertical foram maiores na categoria juvenil, fato esperado tendo em vista a maior idade. AlmuZAInI (2007) relata alta correlação entre idade, força e potência. O autor usou "handgrip" e salto vertical, também utilizados no presente trabalho, entre outras variáveis, para descrever características funcionais de crianças e adolescentes sauditas. Considerando que o IMC dos velejadores juvenis foi similar ao da categoria infantil, sugere-se que a massa corporal da categoria juvenil seja mais efetiva na produção de força e potência.

Em crianças não atletas do sexo masculino com idade entre 11 a 14 anos, Esteves, Leite, Stolt, Sagawa Junior, Borges, Gomes, Santos e Piucco (2005) verificaram o aumento lento e progressivo da força de preensão manual, com intervalos maiores aos 11 - 12 anos, e abrupto na fase púbere, de 13 a 14 anos. Tais resultados corroboram com os achados desse estudo. Os valores médios de força de preensão manual, relatados por EsTEVES et al. (2005), para crianças e adolescentes não atletas, foram menores 
que os obtidos no presente estudo, considerando a mesma faixa etária, o que era esperado numa comparação entre atletas e não atletas, em estudo conduzido no mesmo país. Os não atletas sauditas também obtiveram menor força de preensão e altura de salto vertical. A força de preensão medida em escaladores juvenis (WATTS et al., 2003) superou a mensurada nos velejadores neste estudo, provavelmente devido à valência do treinamento e da prática desportiva da escalada.

\title{
Conclusão
}

O presente estudo apresentou algumas características antropométricas e funcionais de atletas velejadores $\mathrm{da}$ classe "Optimist", que participaram do Campeonato Estadual do Rio de Janeiro. Os resultados demonstram que os velejadores da categoria juvenil apresentaram característica longilínea, sendo mais altos e mais fortes que os da categoria infantil, porém com menor $\% \mathrm{G}$ e massas similares. Os resultados permitiram analisar diferenças entre crianças e jovens praticantes de vela, de outros esportes e populações de não atletas, a partir dos parâmetros levantados e de dados antropométricos e funcionais relatados na literatura.

\begin{abstract}
Anthropometric and functional characteristics of optimist dinghy sailors

This study presents an anthropometric and functional descriptive analysis of young Optimist dinghy sailors, competitors of the Rio de Janeiro State Sailing Championship, in 2006. There were evaluated 50 male sailors, 17 from the infant category and 33 from the junior category. Infant category sailors presented the following descriptive values (mean values \pm standard deviation): $152.8 \pm 5.8 \mathrm{~cm}$ of height, $45.6 \pm$ $5.5 \mathrm{~kg}$ of body mass, $19.6 \pm 2.8 \mathrm{~kg} / \mathrm{m}^{2}$ of BMI, and $23.9 \pm 7.0 \%$ of body fat. Regarding the functional characteristics, handgrip strength was $22.5 \pm 2.9 \mathrm{kgf}$ and vertical jump height was $25.5 \pm 4.8 \mathrm{~cm}$. Junior category had mean stature of $161.7 \pm 7.7 \mathrm{~cm}$, body mass of $48.9 \pm 7.5 \mathrm{~kg}, \mathrm{BMI}$ of $18.7 \pm 2.2 \mathrm{~kg} / \mathrm{m}^{2}$, fat percentage of $18.6 \pm 5.5 \%$, handgrip strength of $29.6 \pm 5.5 \mathrm{kgf}$ and mean vertical jump height of 31.6 $\pm 4.7 \mathrm{~cm}$. Junior category was significant taller and stronger than Infant category $(p<0.05)$. However, the fat percentage was significant lower, resulting in similar BMI and body mass between categories. The results indicate that Junior sailors have a thinner body profile, higher handgrip strength and height are followed by a reduction of the percentage of body fat and maintenance of body mass within limits that are not harmful to the athlete's performance in Optimist boats.
\end{abstract}

UnITERMs: Anthropometric; Optimist; Infants; Adolescents; Sailors.

\section{Referências}

ALMUZAINI, K.S. Muscle function in saudi children and adolescents: relationchip to anthropometric characteristics during growth. Pediatric Exercise Science, Champaign, v.19, p.319-33, 2007.

BAXTER-JONES, A.D.; HELMS, A.D.; MAFFULLI, N.; BAINES-PREECE, J.C.; PREECE, M. Growth and development of male gymnasts swimmers, soccer and tennis players: a longitudinal study. Annals of Human Biology, Basingstoke, v.22, p.381-94, 1995.

BÖHME, M.T.S. O Treinamento a longo prazo e o processo de detecção, seleção e promoção de talentos esportivos.

Revista Brasileira de Ciências do Esporte, Porto Alegre, v.21, p.4-10, 2000.

BOILEAU, R.A.; LOHMAN, T.G.; SLAUGHTER, M.H. Exercise body composition in children and youth. Scandinavian Journal of Sports Sciences, Helsinki, v.7, p.17-27, 1985. 
CACCIARI, E.; MAZZANTI, L.; TASSINARI, D.; BERGAMASCHI, R.; MAGNANI, C.; GHINI, T.; TANI, G.; DRAGO, E.; NANNI, G.; COBIANCHI, C. Growth and sport. Journal of Endocrinological Investigation, Milano,v.12, p.53-7, 1989.

DAMSGAARD, R.; BENCKE, J.; MATTHIESEN, G.; PETERSEN, J.H.; MÜLLER, J. Body proportions, body composition and pubertal development of children in competitive sports. Scandinavian Journal of Medicine \& Science in Sports, Copenhagen, v.11, p.54-60, 2001.

ESTEVES, A.C. ; LEITE, R.M.; STOLT, L.R.O.G. ; SAGAWA JUNIOR, Y.; BORGES J.R.; GOMES, N.; SANTOS, S.G.; PIUCCO, T. Avaliação da força de preensão manual em crianças de 7 a 14 anos.. In: CONGRESSO BRASILEIRO DE BIOMECÂNICA, 11., 2005, João Pessoa. Anais... João Pessoa: SBB, 2005.

FARIAS, E.S.; SALVADOR, M.R.D. Antropometria, composição corporal e atividade física de escolares. Revista Brasileira de Cineantropometria e Desempenho Humano, Florianópolis, v.7, p.21-9, 2005.

FEDERAÇĀO BRASILEIRA DE VELA E MOTOR, 2008. Disponível em: <http://www.maresbrazil.com/>. Acesso em: 4 abr. 2008.

GELADAS, N.D.; NASSIS, G.P.; PAVLICEVIC, S. Somatic and physical traits affecting sprint swimming performance in young swimmers. International Journal of Sport and Medicine, Stuttgart, v.26, p.139-44, 2005.

HELMUTH, H.S. Anthropometric survey of young swimmers. Anthropology Anz, Stuttgart, v.38, p.17-34, 1980.

LEONE, M.; LARIVIERE, G.; COMTOIS, A. S. Discriminant analysis of anthropometric and biomotor variables among elite adolescent female athletes in four sports. Journal of Sports Science, London, v.20, p.443-49, 2002.

PRESTES, J.; LEITE, R.D.; LEITE, G.S.; DONATTO, F.F.; URTADO, C.B.; BARTOLOMEU NETO, J.; DOURADO, A.C. Características antropométricas de jovens nadadores brasileiros do sexo masculino e feminino em diferentes categorias competitivas. Revista Brasileira de Cineantropometria e Desempenho Humano, Florianópolis, v.8, p.25-31, 2006.

REILLY, T.; BANGSBO, T.; FRANKS, A. Anthropometric and physiological predispositions for elite soccer. Journal of Sports Science, London, v.18, p.669-83, 2000.

ROESLER, H.; HAUPENTHAL, A.; SCHUTZ, G.R.; MENEZES, F.S. Método de instrumentação para análise de movimentos de velejadores da classe laser. In: CONGRESSO BRASILEIRO DE BIOMECÂNICA, 9., 2005, João Pessoa. Anais... João Pessoa: SBB, 2005.

SCHNEIDER P.; MEYER, F. Avaliação antropométrica e da força muscular em nadadores pré-púberes e púberes. Revista Brasileira de Medicina do Esporte, São Paulo, v.11, p.209-13, 2005.

TAN, B.; SUNARJA, F. Body mass changes and nutrient intake of optimist class sailors on a race day. Journal of Sports Science, London, v.25, p.1137-40, 2007.

VIVIANI, F.; CASAGRANDE, G.; TONIUTTO, F. The morphotype in a group of peri-pubertal soccer players. Journal of Sports Medicine and Physical Fitness, Torino, v.33, p.178-83, 1993.

WATTS, P.B.; JOUBERT, L.M.; LISH, A.K.; WILKINS, A. Anthropometry of young competitive sport rock climbers.

British Journal of Sports Medicine, London, v.37, p.420-24, 2003.

ENDEREÇO

Adriana Ribeiro de Macedo

Departamento de Fisioterapia - Campus Realengo Instituto Federal de Educação, Ciências e Tecnologia do Rio de J aneiro R. Prof. Carlos Wenceslau, 343 21715-000 - Rio de Janeiro - RJ - BRASIL e-mail: adriana.macedo@ifrj.edu.br
Recebido para publicação: 30/ 04/2008

Revisado: 18/01/2010

Aceito: 17/ 11/ 2010 